\title{
La graphie du dialecte de Surgut du khanty hier et aujourd'hui
}

The Surgut Khanty Alphabet Yesterday and Today

Surguti handi tähestik eile ja täna

\section{Agrafena Pesikova}

Traducteur : Eva Toulouze et Jean-Léo Léonard

\section{(2) OpenEdition \\ Journals}

Édition électronique

URL : https://journals.openedition.org/efo/7111

DOI : $10.4000 /$ efo. 7111

ISSN : 2275-1947

Éditeur

INALCO

Édition imprimée

ISBN : 978-2-343-12463-6

ISSN : 0071-2051

Référence électronique

Agrafena Pesikova, "La graphie du dialecte de Surgut du khanty hier et aujourd'hui ", Études finnoougriennes [En ligne], 48 | 2017, mis en ligne le 20 juillet 2017, consulté le 08 juillet 2021. URL : http:// journals.openedition.org/efo/7111; DOI : https://doi.org/10.4000/efo.7111

Ce document a été généré automatiquement le 8 juillet 2021.

\section{(c) (i) (8)}

Études finno-ougriennes est mis à disposition selon les termes de la Licence Creative Commons Attribution - Pas d'Utilisation Commerciale 4.0 International. 


\title{
La graphie du dialecte de Surgut du khanty hier et aujourd'hui
}

\author{
The Surgut Khanty Alphabet Yesterday and Today \\ Surguti handi tähestik eile ja täna
}

Agrafena Pesikova

Traduction : Eva Toulouze et Jean-Léo Léonard

1 Depuis le début des années 1990, on a assisté à un débat sur la représentation graphique des sons des dialectes septentrionaux du khanty. Or pour le dialecte de Surgut du khanty, ce débat n'a pas eu lieu, les chercheurs travaillant sur la phonologie des dialectes orientaux se comptant sur les doigts d'une main. Il existe cependant une graphie pour les dialectes de Surgut, du Vah et du Vasjugan, élaborée en son temps par N.I. Tereškin, docteur en philologie, sur la base de l'alphabet cyrillique. Mis à part quelques avancées dans le domaine de la phonétique et de la graphie du dialecte de Surgut, cette graphie est toujours reconnue par les chercheurs étrangers qui ont travaillé sur les dialectes orientaux du khanty.

2 Dans ses travaux établissant la graphie des dialectes orientaux, N.I. Tereškin est parti des principes de la graphie du russe tout en tenant compte des particularités des langues ougriennes de l'Ob'. Mais il devait tenir également compte des possibilités typographiques de l'Union soviétique. C'est pourquoi son système repose sur deux principes : le principe phonémique et le principe syllabique.

D'après le principe phonémique, à tout phonème doit correspondre un graphème. Le principe syllabique part d'un ensemble de phonèmes au sein d'une syllabe, qui est écrit avec les graphèmes correspondants.

4 De plus, en raison du caractère limité des lettres possibles dans les typographies soviétiques, plusieurs graphèmes représentaient des phonèmes différents, ce dont il sera question plus bas.

5 À l'époque de N.I. Tereškin, le dialecte de Surgut connaissait 42 graphèmes, dont, entre autres, des caractères russes qui ne correspondaient pas à la langue khantye. C'était une contrainte de l'époque, liée à la situation politique du pays. 


\title{
L'alphabet du dialecte de Surgut d'après N.I. Tereškin
}

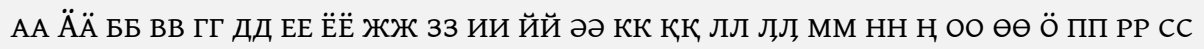 \\ тТ уу ӱў ФФ Х цц чч ччч шш щщ ъ Ыы ь ээ юю яя
}

Selon N.I. Tereškin, l'alphabet du dialecte de Surgut était composé des graphèmes vocaliques, tels que AA Ä̈̈ ЕЕ ЁЁ ии әә оо өө ӧ уу ӱӱ ыЫ ээ юю яя, donc 14 paires de voyelles et ö hors corrélation, donc, en tout, 29 graphèmes; des graphèmes

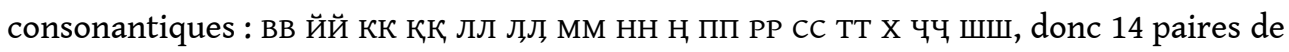
consonnes, ainsi que les graphèmes $\mathrm{H}$ et $\mathrm{x}$ hors corrélation. En tout, 30 graphèmes.

- Graphèmes de signes de ligature, 2 lettres : $\mathrm{b}, \mathrm{b}$.

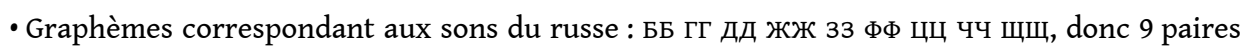
de consonnes.

7 D'après N.I. Tereškin, les phonèmes $\langle A\rangle$ et $\langle\theta\rangle$ correspondaient à $\langle A\rangle,\langle\bar{A}\rangle$ soit $\langle A\rangle,\langle C$ S et $\langle\theta\rangle,\left\langle\left[\begin{array}{ll}10 \\ 0\end{array}\right\rangle\right.$, soit $\langle\ddot{e}\rangle,\langle\hat{e}\rangle$ dans la mesure où la typographie du département de Leningrad des éditions scolaires (Učpedgiz) ne possédait pas de caractère correspondant aux phonèmes $/ \breve{A} /, /$ 员/, / $\breve{y} /$. Les sons vocaliques précédés de yod $\langle\mathrm{A}>$,

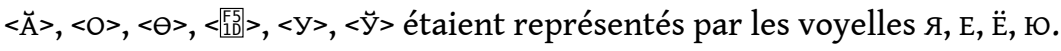

La lettre B représentait aussi bien un son bilabial que la labiodentale $\langle\mathrm{v}>$. La lettre $\mathrm{X}$ également renvoyait à quatre sons, notamment $<\mathrm{X}>$ et $<\$>$.

\begin{tabular}{|c|c|c|c|c|}
\hline AA & $\theta \theta$ & yy & BB & $\mathbf{x}$ \\
\hline $\begin{array}{l}\text { voyelle brève (ou } \\
\text { relâchée) }<\breve{A}>\end{array}$ & $\begin{array}{l}\text { voyelle brève } \\
<\sqrt{15}>>\end{array}$ & voyelle longue $<\breve{y}>$ & $\begin{array}{l}\text { consonne } \\
\text { labiodentale }<\mathrm{B}>\end{array}$ & consonne $<\mathrm{X}>$ \\
\hline HH & TT & EE & Ë̈̈ & Ë̈̈ \\
\hline $\begin{array}{l}\text { consonne }<\mathrm{H}>\text {, } \\
\text { l'apex de la } \\
\text { langue touchant } \\
\text { les alvéoles }\end{array}$ & $\begin{array}{lr}\text { consonne } \quad<\mathrm{T}> \\
\text { l'apex de } \mathrm{la} \\
\text { langue touchant } \\
\text { les alvéoles }\end{array}$ & $\begin{array}{l}\text { voyelle <э> après } \\
\text { consonnes }\end{array}$ & $\begin{array}{l}\text { voyelle }<\theta>\text { après } \\
\text { consonnes } \\
\text { coronales }<\mathrm{Hb}>\text {, } \\
<\mathrm{Tb}>\end{array}$ & $\begin{array}{l}\text { voyelle }<\Theta>\text { après } \\
\text { consonne } \\
\text { médiopalatale } \\
<\breve{\text { }>}\end{array}$ \\
\hline $\begin{array}{lr}\text { consonne } & <\mathrm{H}>\mathrm{du} \\
\text { russe, } & \text { l'apex } \\
\text { touchant } & \text { les } \\
\text { dents } & \end{array}$ & $\begin{array}{lr}\text { consonne } & <\mathrm{T}>\mathrm{du} \\
\text { russe, } & \text { l'apex } \\
\text { touchant } & \text { les } \\
\text { dents } & \end{array}$ & $\begin{array}{l}\text { voyelle <э> après } \\
\text { consonne } \\
\text { médiopalatale <Й> }\end{array}$ & $\begin{array}{l}\text { voyelle }<[15>\text { après } \\
\text { consonnes } \\
\text { palatales < } \\
<\mathrm{Tb}>\end{array}$ & $\begin{array}{l}\text { voyelle }\left\langle\left[\begin{array}{l}{[51} \\
\text { consonne }\end{array}\right.\right. \\
\text { conès } \\
\text { médiopalatale } \\
<\breve{\text { }}>\end{array}$ \\
\hline юю & юю & ии & яя & яя \\
\hline $\begin{array}{l}\text { voyelle }<\mathrm{y}>\text { après } \\
\text { consonne } \\
\text { médiopalatale } \\
<\breve{\text { > }>}\end{array}$ & $\begin{array}{l}\text { aussi }<\breve{y}>\text { après } \\
\text { consonne } \\
\text { médiopalatale } \\
<\breve{И}>\end{array}$ & $\begin{array}{l}<\mathrm{l}>\text { après consonnes } \\
\text { sauf prépalatales } \\
<\mathrm{H}>, \quad<\mathrm{T}>\text { après } \\
\text { lesquelles on notait } \\
\mathrm{b}\end{array}$ & voyelle <A> & $\begin{array}{l}<\breve{A}>\quad \text { après } \\
\text { consonne } \\
\text { médiopalatale } \\
<\breve{\text { }}>\end{array}$ \\
\hline
\end{tabular}




\begin{tabular}{|c|c|c|c|c|}
\hline $\begin{array}{l}\text { voyelle }<\mathrm{y}>\text { après } \\
\text { consonnes } \\
\text { médiopalatales } \\
<\mathrm{Hb}>,<\mathrm{Tb}>\end{array}$ & $\begin{array}{l}<\breve{y}>\quad \text { après } \\
\text { consonne } \\
\text { médiopalatale } \\
<\breve{\text { }>}\end{array}$ & $\begin{array}{l}<И>\text { après toutes } \\
\text { consonnes dans les } \\
\text { mots empruntés au } \\
\text { russe }\end{array}$ & $\begin{array}{l}\text { voyelle }<\mathrm{A}>\text { après } \\
\text { consonnes } \\
\text { médiopalatales } \\
<\mathrm{Hb}>,<\mathrm{Tb}>\end{array}$ & $\begin{array}{l}\text { voyelle }<\breve{A}>\text { après } \\
\text { consonnes } \\
\text { médiopalatales } \\
<\mathrm{Hb}>,<\mathrm{Tb}>\end{array}$ \\
\hline
\end{tabular}

Comme nous le voyons dans ce tableau, les graphèmes $\mathbf{A}, \Theta$, и renvoient chacun à deux sons. C'est le cas également du E. Quant aux lettres $\ddot{E}, \mathfrak{ю , ~}$, , on les trouve dans quatre valeurs différentes.

Aujourd'hui, la notation cyrillique des sons du dialecte de Surgut du khanty a quelque peu changé. D’après la théorie de Márta Csepregi (que je partage), les graphèmes devraient être :

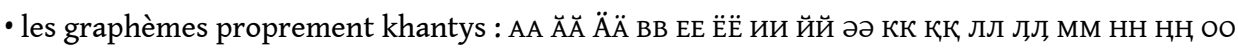

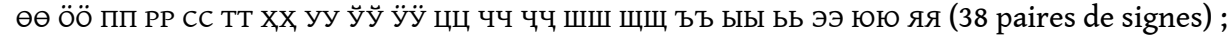

• les lettres : ББ гг дд жж $3 з$ ФФ ХХ цц empruntées au russe (8 paires de signes).

\section{Correspondances actuelles entre les sons et les lettres}

11 Le phonème / $\mathrm{A} /$ correspond à un son long ou tendu, il s'écrit $\mathrm{A}$ en début de mot, ainsi qu'à l'intérieur ou à la fin du mot après toutes consonnes, sauf les consonnes

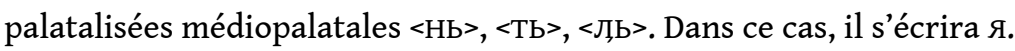

Exemples : нянь «pain », ТяС « montre », Л,яЛЬ « guerre ».

La lettre 9 correspond au phonème $<A>$ en début et fin de mot et après le phonème $<\breve{И}>$.

Exemples : ЯңК « broche », Ясәң « récit », ястәтА « vers le renne ».

13 Le phonème $<\breve{A}>$ est un son court, qui s'écrit $\breve{A}$ au début, à l'intérieur et à la fin du mot, après le phonème $<\breve{И}>$ et les consonnes médiopalatales, palatalisées.

Exemples : ӐвәЛ « traîneau », ЙӐВวH « rivière », НЬĂСTA « glisser », КÖТАM « ma main », кила « lève-toi ! ».

14 Le phonème $<И>$ est un son long qui s'écrit $И$, il est situé à l'avant, à l'intérieur et à la fin du mot et s'associe avec toutes les consonnes: il figure après les labiales $\langle M\rangle,\langle\Pi\rangle,\langle B\rangle$, les prépalatales $\langle\mathrm{T}\rangle,\langle\amalg>,\langle\mathrm{C}\rangle,\langle\pi\rangle,\langle\pi\rangle,\langle\mathrm{H}\rangle,\langle\mathrm{P}\rangle$, les médiopalatales $\langle\breve{\mathrm{h}}\rangle,\langle\mathrm{\Psi}\rangle,\langle\pi, \mathrm{h}\rangle$, $\langle\mathrm{Hb}\rangle,\langle\mathrm{Hb}\rangle,\langle\mathrm{Tb}\rangle$, les postlinguales $\langle\mathrm{K}\rangle,\langle\mathrm{K}\rangle,\langle\mathrm{H}\rangle,\langle\mathrm{X}\rangle$.

Exemples: иңәл "ébréchure », ими «femme ", мис "traîneau de charge ", кирип «bateau », сохит « le long de », қури « cuve », ЙимәлৃТА « se couvrir le visage ».

15 La notation du phonème $\langle h>$ répond à certaines règles orthographiques avec les consonnes prépalatales $\langle\pi\rangle,\langle\pi\rangle,\langle\mathrm{H}\rangle,\langle\mathrm{T}\rangle$, et médiopalatales $\langle\Omega \mathrm{b}\rangle, \mathrm{Hb}\rangle,\langle\mathrm{T} b\rangle$. Le problème est que le phonème $<h>$ diffère du phonème russe $<h>$ : en prononçant le son khanty correspondant, la partie centrale de la langue remonte davantage vers le palais que dans la prononciation du son russe $\langle И>$. C'est pourquoi dans la graphie du khanty, la signification de la lettre и diffère de celle de l'équivalent russe. D'après les principes orthographiques de N.I. Tereškin, la lettre И dans le khanty de Surgut peut figurer après les consonnes médiopalatales palatalisées $\langle\mathrm{Hb}\rangle,\langle\mathrm{Tb}\rangle$, alors qu'après les 
prépalatales dures $<\mathrm{H}>,<\mathrm{T}>$ il est prévu d'utiliser, au lieu du $и$, la lettre justifiant par le petit nombre de mots dans lesquels cette combinaison figure.

Exemples : тиминт « tel, telle », НиР « prix ».

Mais il faut écrire non pas $\mathrm{T}<И>\mathrm{H}$, mais тын «prix, valeur », non pas $\mathrm{H}<И>П Е к$ mais НЫПЕК « papier », non pas $\mathrm{H}<И>\mathrm{H}$ mais нын « vous deux ».

Il y a d'autres combinaisons de phonèmes compliquées à écrire, comme celle de $<\int \preceq b$ et de $<$ $\langle$.

Exemples: ЛЬ<И>льтьәХтәтА « réciter une incantation, dire une prière »; ЛЬ<И>Кәм, лৃь<И>Кән, Лூь<И>К « j'ai tiré, tu as tiré, il a tiré » (au fusil).

Ma pratique d'enseignement me montre que les élèves assimilent facilement la combinaison $<\Omega>$ et $\langle$ $\langle$ > :

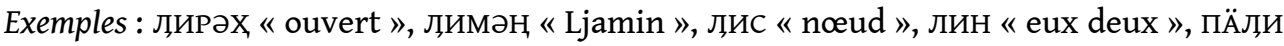
« île surélevée dans un marais ».

19 La situation est bien meilleure avec la combinaison du phonème prélingual dur $\langle\pi\rangle$ et du son $\langle И\rangle$, dans la mesure où le dialecte de Surgut ne connaît pas de paire mouillée pour le phonème $<\pi>$.

Exemples: липәк « renard arctique », лиңкәм «j'ai couvert », пыли « pelle », лимАс «nénuphar ».

20 Le phonème <ы>, une voyelle longue, est noté en toutes positions par la lettre b. Il peut figurer au début et à l'intérieur d'un mot.

Exemples: ЫРАП «méchant», Ытәң «fort», ЫмЛ̧А (pour les boissons), мыЧ «faute, accusation », қытьтя « rester », қырәх «sac», тьыңқитәтА « taquiner, vexer», льыньте « enfonce », пыхәр, чымәЛ « peu ».

21 Ce qui s'écrit b en fin de mot, après les phonèmes $<H>$ et $<\mathrm{T}>$, se prononce $<И>$.

Exemples: қот<И> - қоты «depuis la maison», Рыт<и> - Рыты «de la barque», пын<и> - пыны « depuis la verrue », Т<И>н<И> - тыны « de la valeur ».

Le phonème $<\ni>$ est une voyelle longue; en début de mot ainsi qu'après les consonnes prépalatales $\langle\mathrm{H}\rangle,\langle\mathrm{T}\rangle,\langle\mathrm{C}\rangle,\langle\mathrm{J}\rangle,\langle\mathrm{J}\rangle$ il est marqué par $\ni$.

Exemples : эвәт « mont sacré », нэ « femme », тэми « сесі », нэлиңки « Nelinki » (nom de femme), тэрәмня «village », нэви «blanc », сэл « poisson séché », лэк « route », лฺэв « tourbillon ».

23 Après les consonnes labiales $\langle\Pi>,\langle\mathrm{M}\rangle,\langle\mathrm{B}\rangle$, la bilabiale $\langle\mathrm{P}\rangle$ et les dorsales et postdorsales $\langle\mathrm{K}\rangle,\langle\mathrm{K}\rangle,\langle\mathrm{X}\rangle,\langle\mathrm{H}\rangle$, ce même phonème n'est pas noté $\ni$ mais $\mathrm{E}$.

Exemples: ПЕВ «bosse », МЕВНЫТА «bâiller », ВЕЛ, « renne», РЕВ « cendre», КЕР

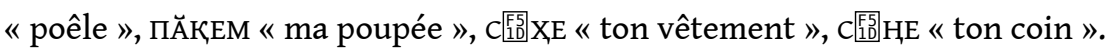

Après les consonnes médiopalatales $\langle\mathrm{Hb}\rangle,\langle\mathrm{Tb}\rangle,\left\langle\int \mathrm{b}\right\rangle,\langle\mathrm{\Psi}\rangle$, le phonème $\ni$ est noté par la lettre $\mathrm{E}$.

Exemples: НЕВРем « enfant », НЕВәР « écume », НЕМАҚ « Nemako » (nom d'homme), ТЕНӘ « ainsi », ЛุЕК « fourré près d'une rivière », ЧุЕК « malheur ».

En combinaison avec le son médiopalatal $<\breve{И}>$, le phonème $<\ni>$ se note $E$.

Exemples : еК « danse », Ең « dix », ЕНәК « banlieue », ВУЕм «j'ai vu ( (conjugaison non objective), BəЕМ « j’ai pris » (conjugaison non objective), С̈̈ЕM « mon bruit ». 


\section{Exemples : Й $<\Theta$
ТЁРИ « seau ».} neige $»$. «j'ai rongé ». consonnes.

Le phonème $<y>$ est une voyelle longue; en début de mot et après les consonnes prépalatales dures $\langle\mathrm{H}\rangle,\langle\mathrm{T}\rangle,\langle\mathrm{C}\rangle,\langle\mathrm{J}\rangle,\langle\mathrm{J}\rangle,\langle\mathrm{U}\rangle$, ainsi qu'après les labiales $\langle\Pi\rangle,\langle\mathrm{M}\rangle,\langle\mathrm{B}\rangle$, la prélinguale $<\mathrm{P}>$ et les (post)dorsales $<\mathrm{K}>,<\mathrm{X}>,<\mathrm{H}>$, il est noté par $\mathrm{y}$.

Exemples : Удңәл « son début », УР « possibilité », НУР « responsabilité », ТУР " gorge », сурты « animal âgé de deux ans », лумП «ski de chasseur», ЛуУ « rame », ПУН «pelage ", МУНТ « récemment », ВУТә « en haut», РУПшәң « ébouriffé », қУЙқ « homme », мәхув «notre terre », пиңув « notre doigt», Чухтәм «ma chute de

Après les consonnes médiopalatales $\langle\mathrm{Hb}\rangle,<\mathrm{Tb}\rangle,<$ Л, $\mathrm{b}\rangle$, le phonème y est noté $Ю$.

Exemples : НюР « peau », ТюРСэм « intérêt », ЛюЛЬәм « je me suis levé », Тюмәм « ma possibilité », Тюнь « chance abondante », Тюти « sein pendant l'allaitement ».

Le phonème $<y>$ combiné avec la médiopalatale $<\breve{И}>$ s'écrit aussi

Exemples: Юмпәң « aérien », юПлиптА « faire preuve de mauvais goût », вәйхиюв « nous avons essayé de prendre », МәнХиюв « nous avons essayé de partir », ЮңқРәМ

Le phonème $<0>$ est une voyelle longue, notée $\mathrm{O}$ en début de mot et après toutes les

Exemples: оләң « début», омәстА «être (assis)», нохәр « pigne de cèdre », сос «hermine», мой «demande en mariage», қор «taureau», поңқ « amanite tuemouches », тоРәХ « cigogne», лот « endroit», ЛОР « lac», ЙоХ « gens », Тьом " possibilité », НьохәЛ « bas », чопәҚҚА « vérité », ЛЬоньть « neige ».

Pour les mots empruntés au russe, N.I. Tereškin, dans les manuels du dialecte de Surgut du khanty, utilise la lettre $o$, bien que le son ainsi désigné présente des caractéristiques différentes. Rappelons que le phonème $<0>$ du dialecte de Surgut se prononce à la racine de la langue, et occupe une position intermédiaire entre $<A>$ et $<\theta>$ sans que les lèvres s'arrondissent, alors qu'en russe elles s'arrondissent.

Le mot «kolkhoze » est prononcé : КӐлкөс par les Khantys de Surgut. Mais dans les manuels il s'écrit колхоз.

Le phonème $\langle\theta>$ est une voyelle longue et figure en début de mot et après toutes les consonnes à l'exception des consonnes médiopalatales $<\breve{h}>,<\mathrm{Hb}>,<\mathrm{Tb}>$; il est noté par $\theta$. Il se prononce $<\theta>$, les lèvres sont arrondies.

Exemples: $ө$ « « embouchure », Өңқ « résine », қөР «trouée (corridor) », Нөмән « еn haut », мөқ « petit», лөттА « acheter », төппә « seulement», төли « Teli » (nom d'homme), ЛөнТ « oie ».

3 Après les consonnes médiopalatales $\langle\mathrm{Hb}>,<\mathrm{Tb}\rangle,<\breve{h}>$ le son $<\theta>$ est noté par la lettre Ë.

Le phonème $<[10]>$ est une consonne brève, qui en début de mot et après toutes les consonnes est notée par la lettre [1.5. $_{10}$.

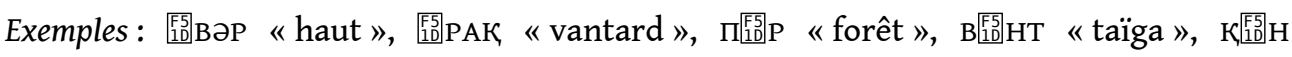
« ventre », Л!

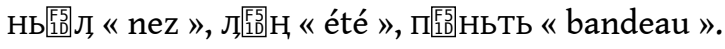


Les phonèmes $<\ddot{A}>$ et $<\ddot{y}>$ sont des voyelles ultrabrèves, qui sont notées respectivement $\ddot{A}, \ddot{y}$ en début de mot et après toutes les consonnes.

Exemples: ӓпәл « odeur», ӒВи «petite fille», ӱлтА «chauffer (le poêle), ӱлАк «bretelle », тёвләксәтА « se promener dans le noir », пёңк « dent », тӥләх « hiver », пӱки « confident », ЛуЎЙ « pus », РӱТ « fils qui tombent quand on coupe un tissu », ЛЬ ̈̈в « neveu », ТЬӰкНәң « courbe », ЙӒВ « perche (poisson)», ЙӰТА « arriver (à pied)».

Le phonème $<\ddot{O}>$ est une voyelle ultrabrève qui ne se rencontre qu'à l'intérieur d'un mot et qui est notée par la lettre ö.

Exemples : кӧт « main », вӧң « gendre », вӧв « force », ЧӒңкәЛТА « devenir adulte », кӧпәл « revêche ».

Les phonèmes extra-courts prélinguaux $<\ddot{\mathrm{A}}>,<\ddot{O}>,<\ddot{\mathrm{y}}>$ ne peuvent, en raison de la spécificité de leur prononciation, figurer après les consonnes (post)dorsales $<$ $>,<\mathrm{\text { }}$, $<\mathrm{X}>$.

Les descendants des Khantys du Kazym qui se sont installés définitivement dans le bassin du Tromjogan et qui se considèrent comme appartenant au groupe de cette rivière ne prononcent pas le son $<\ddot{y}>$ et le remplacent par <ə>.

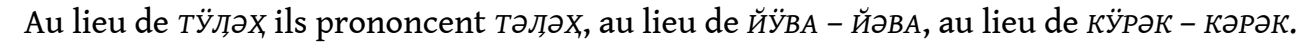

Le phonème <ə> est une voyelle ultrabrève qui peut figurer en début, à l'intérieur et en fin de mot, et peut s'associer avec n'importe quelle consonne. Il est noté par la lettre ə. Dans le dialecte de Surgut, c'est le son le plus fréquent.

Exemples : әнтәП « ceinture, taille », ньәлу « quatre », тьәри « nœud », пәтә « fond », गььәки « état d'esprit malveillant», пәхтә « noir », РәкәнтА « s'envoler », сәй « langue de sable », ләХ, « queue ».

Les phonèmes $\langle\mathrm{B}>,\langle\mathrm{M}>,<\Pi>$ sont des consonnes labiales auxquelles correspondent les lettres $\mathrm{B}, \mathrm{M}, \Pi$. Dans le dialecte de Surgut, la lettre $\mathrm{B}$ correspond à deux sons différents : B - bilabial et B - labiodental.

Exemples: В ${ }_{10}^{[5}$ ПУВЛӘМ « joue », ПӐВЛәМТА « se bousculer ».

Mais dans les mots d'emprunt, elle correspond au son $<\mathrm{B}>$ labiodental.

Exemples: ВАННА «bain», РЕВОЛюЦИЯ « révolution», ВАРНАК « prisonnier », ЗВОНИТ ЛџТА « résonner ».

43 Les phonèmes $\langle\pi>,<$ Л $>,<H>,<\mathrm{P}\rangle,\langle\mathrm{C}>,<\mathrm{T}>,<\amalg>$ sont des consonnes prépalatales et sont respectivement notés par les lettres Л, Л, Н, Р, С, Т, Ш.

44 Le phonème $<\mathrm{H}>$ diffère $\mathrm{du}$ son russe $\mathrm{H}$. Si, en russe, ce son est prononcé de telle sorte que le bout de la langue touche les dents, dans le dialecte khanty de Surgut, il touche les alvéoles.

Les phonèmes $\langle\pi>,\langle C>,<\amalg>$ ne connaissent pas de paire mouillée aux phonèmes $<J>$, $<\mathrm{H}>,<\mathrm{T}>$. Les phonèmes $<$ Л, $\mathrm{b}>,<\mathrm{Hb}>,<\mathrm{Tb}>,<\breve{И}>,<\mathrm{\varphi}>$ sont des consonnes médio-linguales respectivement notées par ЛЬ, НЬ, ТЬ, Й, Ч.

Le phonème $<\mathrm{T} b>$ est utilisé dans les parlers du Jugan, du Pim et de l'Agan. Dans le parler du Tromjogan ce phonème se transforme en un son mouillé <५>. 
Exemples : ПәтЯ - ПәЧА « bonjour », ТЕНӘ - ЧЕНӘ « ainsi », ТЬЫңқИТӘТА - ЧИҢҚИТәТА « taquiner », ТЬорәС - чорәС « mer », ТЬ医

\section{ultrabrèves $\langle\ddot{\mathrm{A}}\rangle,\langle\partial>$, $<\ddot{\mathrm{O}}>$.}

Exemples : пёңкләК « colonne vertébrale », СәНКЕ « bats ! », ВӦНӘН « ton gendre ».

Exemples : КӒВИ « coucou », КӦТ « main », КӦЛ « mot », КӒККวҢ « pierreux ».

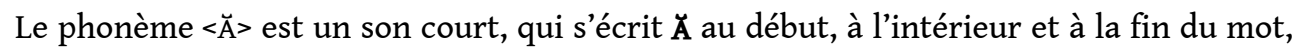
après le phonème $<\breve{И}>$ et les consonnes médiopalatales, palatalisées.

Exemples : ӐВәЛ « traîneau », ЙӐВән « rivière », НЬӐСТА « glisser ».

\section{Conclusion}

J'ai fondé mes travaux concernant la phonétique et la graphie du dialecte de Surgut du khanty sur les œuvres de N.I. Tereškin. Tout au long des années, j'ai moi-même identifié quelques sons prononcés par mes conationaux. Dans les années 1990, j’ai donné des cours aux enseignants de khanty et je suis intervenue également sur ces questions devant des étudiants hongrois. Mais des atermoiements bureaucratiques ne me permettent pas de publier mes recherches dans le domaine de la phonétique et de la morphologie du dialecte de Surgut de la langue khantye. Jusqu'ici, ces travaux sont à l'état de manuscrits. 
Tableau de la graphie contemporaine du dialecte de Surgut du khanty

\begin{tabular}{|c|c|c|c|c|}
\hline AA & Ă & $\ddot{A} A ̈$ & BB & EE \\
\hline \multirow{2}{*}{$\begin{array}{l}\text { Voyelle longue } \\
<A>\end{array}$} & \multirow{2}{*}{ Voyelle brève $<\breve{A}>$} & \multirow{2}{*}{$\begin{array}{l}\text { Voyelle } \\
\text { ultrabrève < }>\text { A }\end{array}$} & $\begin{array}{l}\text { Son bilabial } \\
<\mathrm{B}>\end{array}$ & $\begin{array}{ll}<\ni> & \text { après } \\
\text { consonnes } & \end{array}$ \\
\hline & & & $\begin{array}{l}\text { Son labiodental } \\
<B>\end{array}$ & <ЙЭ> \\
\hline ËË & ии & ЙЙ & วə & КК \\
\hline $\begin{array}{l}\text { Son }\langle\theta>\text { après } \\
\text { médio-linguale } \\
<\breve{И}>\end{array}$ & 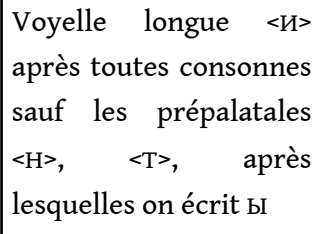 & \multirow{2}{*}{ 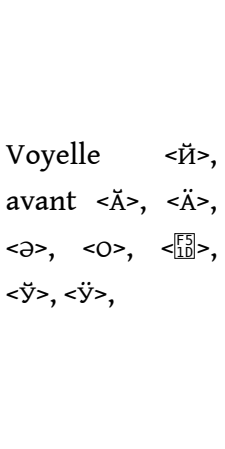 } & \multirow{2}{*}{$\begin{array}{l}\text { Voyelle } \\
\text { ultrabrève <ə>, } \\
\text { avant et après } \\
\text { toute consonne }\end{array}$} & $\begin{array}{l}\text { Consonne dorsale } \\
\text { superficielle }<\mathrm{K}>\end{array}$ \\
\hline $\begin{array}{l}\text { voyelle }<\theta>\text { après } \\
\text { médio-linguales } \\
<\mathrm{Hb}>,<\mathrm{Tb}>\end{array}$ & $\begin{array}{l}\text { Marque la voyelle <и> } \\
\text { du russe, le bout de la } \\
\text { langue touchant les } \\
\text { dents }\end{array}$ & & & \\
\hline ққ & лл & गлЛ & MM & HH \\
\hline \multirow[b]{2}{*}{$\begin{array}{l}\text { consonne } \\
\text { uvulaire <қ> }\end{array}$} & consonne <Л> & \multirow{2}{*}{$\begin{array}{l}\text { consonne } \\
\text { latérale sourde } \\
<\text { ग > }\end{array}$} & \multirow[b]{2}{*}{ consonne $<\mathrm{M}>$} & Consonne $<\mathrm{H}>$ \\
\hline & $\begin{array}{l}\text { Consonne }<Л>\text { dans les } \\
\text { emprunts au russe }\end{array}$ & & & $\begin{array}{l}\text { consonne } \quad<\mathrm{H}> \\
\text { dans les emprunts } \\
\text { au russe }\end{array}$ \\
\hline НH & 00 & $\theta \theta$ & 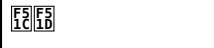 & öö \\
\hline $\begin{array}{l}\text { sonante dorsale } \\
<\mathrm{H}>\end{array}$ & voyelle longue <0> & $\begin{array}{l}\text { voyelle longue } \\
<\Theta>\end{array}$ & $\begin{array}{l}\text { voyelle brève } \\
<\left[\begin{array}{l}150 \\
<1\end{array}\right.\end{array}$ & $\begin{array}{l}\text { voyelle ultrabrève } \\
<0 ̈>\end{array}$ \\
\hline пп & PP & CC & TT & yy \\
\hline \multirow[b]{2}{*}{ consonne $<\Pi>$} & \multirow[b]{2}{*}{ consonne $<\mathrm{P}>$} & \multirow[b]{2}{*}{ consonne $<C>$} & consonne $<\mathrm{T}>$ & $\begin{array}{l}\text { voyelle longue } \\
<y>\end{array}$ \\
\hline & & & $\begin{array}{lr}\text { consonne } & <\mathrm{T}> \\
\text { dans } & \text { les } \\
\text { emprunts } & \text { au } \\
\text { russe } & \end{array}$ & \\
\hline ўЎ & $\ddot{y} \ddot{y}$ & $x x$ & ५५ & ૫૫ \\
\hline Voyelle brève $<\breve{y}>$ & Voyelle ultrabrève <ÿ> & $\begin{array}{l}\text { Consonne } \\
\text { postlinguale } \\
\text { profonde <X> }\end{array}$ & $\begin{array}{l}\text { Consonne } \\
\text { mouillée <५> }\end{array}$ & $\begin{array}{l}\text { Consonne } \\
\text { dure<प > }\end{array}$ \\
\hline
\end{tabular}




\begin{tabular}{|c|c|c|c|c|}
\hline Шш & $\mathrm{b}$ & ыы & b & ээ \\
\hline \multirow[t]{2}{*}{$\begin{array}{l}\text { Consonne } \\
\text { chuintante <ш> }\end{array}$} & Signe dur séparateur & $\begin{array}{l}\text { Voyelle longue } \\
<\mathrm{D}>\end{array}$ & $\begin{array}{l}\text { Signe mou } \\
\text { séparateur }\end{array}$ & $\begin{array}{l}\text { Consonne longue } \\
<\ni>\end{array}$ \\
\hline & & $\begin{array}{l}\text { Voyelle }<\text { }> \\
\text { après consonnes } \\
\text { prépalatales } \\
<\mathrm{H}>,<\mathrm{T}>\end{array}$ & $\begin{array}{l}\text { Mouillure des } \\
\text { médio- } \\
\text { linguales <Hb>, } \\
<\mathrm{Tb}>\end{array}$ & \\
\hline юю & яя & ББ & IT & дд \\
\hline 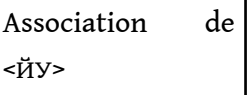 & 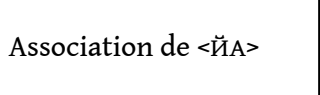 & \multirow[b]{2}{*}{$\begin{array}{l}\text { Consonne } \\
\text { sonore <B> }\end{array}$} & \multirow[b]{2}{*}{$\begin{array}{l}\text { Consonne } \\
\text { sonore }<\Gamma>\end{array}$} & $\begin{array}{l}\text { Consonne sonore } \\
<Д>\end{array}$ \\
\hline $\begin{array}{l}<\mathrm{y}>\quad \text { après } \\
\text { consonnes } \\
\text { médiopalatales } \\
<\mathrm{Hb}>,<\mathrm{Tb}>\end{array}$ & $\begin{array}{l}<\mathrm{A}>\text { après consonnes } \\
\text { médiopalatales <Hb>, } \\
<\mathrm{T} b>\end{array}$ & & & \\
\hline ЖЖ & 33 & $\Phi \Phi$ & $\mathbf{x x}$ & цщ \\
\hline $\begin{array}{l}\text { Consonne sonore } \\
<Ж>\end{array}$ & Consonne sonore $<3>$ & consonne $<\Phi>$ & consonne $<X>$ & consonne <Ц> \\
\hline \multicolumn{5}{|l|}{ щщ } \\
\hline consonne <щ> & & & & \\
\hline
\end{tabular}

On voit dans ce tableau que les lettres B, $\mathbf{~}, \mathbf{H}, \mathbf{T}$ ont deux valeurs : en tant que sons à proprement parler khantys et en tant que sons du russe dans les mots d'emprunt.

57 Toutes les voyelles palatales $\mathbf{E}, \ddot{\mathbf{E}}, \mathfrak{\wp}, \boldsymbol{я}$ jouent un double rôle : d'une part elles marquent la mouillure de la consonne, d'autre part elles marquent l'association de la consonne médio-linguale Й avec les voyelles A, э, $\Theta, \mathbf{y}$.

Par exemple: on prononce ПЕВ, et non ПэВ « pomme de pin »;

ВЕлИ, et non Вэли « renne »;

KEHЯP, et non КЭНЯР « pauvre »;

МЕМИ, et non МэмИ « ours »;

PEB, et non PЭB, « cendre »;

KEP, et non KэP « poêle, four ».

пәтяР « sorbier », тЁНқәЛТәТА « goûter », НЕВРЕм « enfant », НюкИ « emplacement d'une tente », моньтят « avec un conte », тюты « et ainsi ».

Ainsi, nous aboutissons à cet alphabet à proprement parler khanty de Surgut :

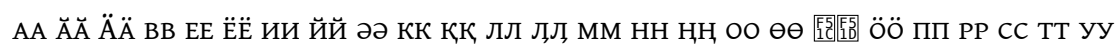

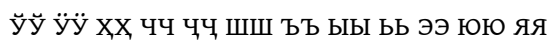

Lettres empruntées au russe :

ББ гг дд жж 33 ФФ XX цц щщ. 
Ainsi, 37 paires de signes représentent les sons originels du dialecte de Surgut, alors que 9 paires de signes représentent les lettres empruntées au russe, ce qui fait en tout 46 paires de signes.

61 Tous les manuels de l'époque soviétique sont écrits dans la graphie proposée en son temps par N.I. Tereškin.

\section{Problèmes de la diffusion du dialecte de Surgut dans les établissements d'enseignement des « raïon » de Surgut, Nižnevartovsk et Neftejugansk}

Depuis 1996, tous les matériaux d'enseignement, la littérature, les pages spéciales du journal Hanty jasan ${ }^{1}$ sont écrits dans la langue des Khantys de Surgut avec les lettres

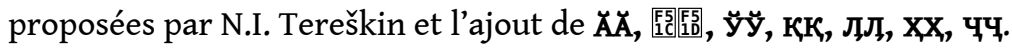

C'est en 2001 seulement qu'avec l'aide de l'informaticien de «Barsova gora » (Surgut, directeur N.V. Šatunov) Maksim Popadkin, a été installé un programme de polices pour le dialecte de Surgut du khanty dans l'ordinateur de la filiale de Surgut de l'Institut ob-ougrien de recherches appliquées, puis dans l'ordinateur de « Hanty jasan ».

Depuis 2010, la police PT Serif figure parmi les polices informatiques. Elle a facilité considérablement la transcription des textes de tradition orale, et a permis de composer des manuels et des œuvres littéraires dans la langue des Khantys de Surgut.

Mais elle n'a pas permis de résoudre les sempiternels problèmes que pose la diffusion du dialecte de Surgut dans les établissements des raïon de Surgut, Nižnevartovsk et Neftejugansk, où habitent traditionnellement les Khantys de Surgut. Ceux-ci utilisent leur langue maternelle non seulement dans la vie de tous les jours, mais dans les institutions officielles, telles que les administrations des raïon et des zones rurales, les tribunaux, les organes de la justice, de la culture (musées, bibliothèques, centres de loisirs, centres artistiques), les écoles-internats. Sur la base d'une enquête menée par l'Institut ob-ougrien auprès des autochtones sur la connaissance de leur langue maternelle, plus de $90 \%$ des Khantys de Surgut déclarent parler leur dialecte. Nous mentionnerons ici quelques-uns des problèmes rencontrés :

1. L'absence de nouveaux manuels et dictionnaires dans le dialecte de Surgut du khanty. Il existe bien des ouvrages vieillis sur cette langue (jusqu'à la cinquième classe), des éditions des années 1980-1990. Dans la première décennie du XxI ${ }^{\mathrm{e}}$ siècle, à l'initiative du Département d'éducation et de politique de jeunesse du gouvernement de HMAO-Jugra, ont été encore publiés des manuels standard (pour les classes 1-4) dans la langue des Khantys de Surgut analogues à ceux publiés dans le dialecte de Kazym pour les enfants ne maîtrisant pas leur langue maternelle. Dans une situation où $90 \%$ de la population utilise quotidiennement la langue maternelle, je considère que la publication de ces manuels pour débutants n'était pas pertinente.

Il est scientifiquement prouvé de nos jours que chaque groupe de dialectes du khanty est une langue à part, avec sa composition phonétique particulière, ses formes grammaticales, sa sémantique propre. Il est pratiquement impossible de parvenir à une unification. Les manuels disponibles dans les différents dialectes illustrent bien cette absence de correspondances. Les dialectes de l'Est sortent des limites du système des dialectes du Nord. Par exemple, les abécédaires des dialectes de Surgut et du Vah ne peuvent guère être construits sur le modèle des manuels pour le dialecte de Šuryškar. La grammaire de la langue des Khantys de l'Est ne peut être formatée dans le système de la langue des Khantys 
du Nord. C'est pourquoi les auteurs des manuels standard ne sont pas satisfaits de cet ensemble d'ouvrages pour les quatre premières classes en dialecte de Surgut, construits sur le modèle des manuels de V. N. Solovar en khanty de Kazym. Nous considérons que les manuels de Valentina Nikolaevna sont excellents pour la langue concernée, mais que son modèle ne convient pas au dialecte de Surgut.

2. L'Institut ob-ougrien de recherches appliquées travaille sous l'égide du Département d'éducation et de politique de la jeunesse et, qu'il le veuille ou non, joue le rôle d'un institut de développement de l'éducation à part entière. Cette situation a des aspects à la fois positifs et négatifs.

1. Elle est positive, dans la mesure où les personnes qui maîtrisent la linguistique khantye et les auteurs des manuels et des dictionnaires en khanty travaillent pour l'essentiel dans l'institut susnommé et non pas dans l'Institut pour le développement de l'éducation.

2. Elle est négative, dans la mesure où l'Institut ob-ougrien a ses objectifs et ses missions : étudier la langue, l'économie, la culture, l'ethnographie des peuples autochtones, alors que l'Institut pour le développement de l'éducation a d'autres objectifs, qui sont de résoudre les problèmes qui se posent dans l'éducation et dans les établissements d'éducation. De plus, ce dernier institut ne dispose pas suffisamment de cadres qualifiés, capables d'éditer des manuels et des dictionnaires dans les dialectes khantys orientaux; il ne dispose pas non plus de résultats relatifs aux études sur les dialectes du Vah et de Surgut.

3. Par ailleurs, un autre facteur a des effets négatifs sur la diffusion des dialectes de l'Est dans la pratique: dans son activité, le Département de l'éducation et de la politique de jeunesse préfère miser sur les dialectes du Nord du khanty, négligeant les dialectes de l'Est dans les écoles et les établissements d'enseignement supérieur.

3. L'absence de cadres compétents concernant les dialectes orientaux. Sur la base des cours de phonétique et de morphologie du dialecte de Surgut que j'ai donnés à Budapest au début des années 1990, Márta Csepregi, docteur en linguistique, a édité en hongrois toute une série de travaux sur le dialecte de Surgut, et a formé 14 spécialistes qui, non seulement connaissent ce dialecte en théorie, mais qui, en outre, parlent fort bien cette langue. Si le Département, l'université d'Iougrie et l'Institut de développement de l'éducation ne parviennent pas à faire face à leur mission, je propose de travailler avec les Hongrois spécialistes de la langue des Khantys orientaux. Ces trois établissements n'ont pas pour mission (nous le savons) de former des cadres compétents sur ces dialectes. De plus, l'université de Iougrie a réduit de manière générale ses travaux sur les langues ougriennes de l'ob'.

4. L'Institut ob-ougrien n'est pas en mesure de travailler à implanter les dialectes orientaux dans la pratique des autochtones pour toute une série de raisons :

1. La première est la spécialisation étroite non seulement de chaque section de l'institut, mais également des personnes qui y travaillent. Par exemple, un spécialiste en langue n'a pas le droit de collecter des matériaux ethnographiques; une personne qui maîtrise bien l'écrit dans son dialecte mais qui n'est officiellement diplômée que de l'enseignement secondaire n'est pas autorisée à transcrire les textes de tradition orale de ses proches.

2. L'absence de moyens techniques modernes. Si tant est qu'il y en ait dans les filiales de l'Institut ob-ougrien, ce sont des anciens modèles, incapables de faire face aux tâches que s'est fixées l'institut, par exemple, la numérisation des enregistrements audio et vidéo ou le transfert de matériaux d'un informateur à un autre. À la filiale de Surgut, il n'existe qu'un seul ordinateur, un modèle de 1998. Il va de soi que cet équipement ne permet pas de composer un manuel de qualité pour le dialecte de Surgut.

5. L'absence de budget pour former des cadres spécialistes des dialectes orientaux du khanty. Sans la participation des institutions de l'État, il n'est guère possible de diffuser les dialectes orientaux dans les établissements d'enseignements des raïon de Surgut, Nižnevartovsk et 
Neftejugansk. Nous avons besoin d'un plan national sur la recherche et l'élaboration de manuels, de livres de lecture, de dictionnaires et d'ouvrages didactiques.

6. Faute de moyens budgétaires, il est pratiquement impossible d'élaborer et d'éditer de nouveaux ouvrages qui n'ont pas vieilli du point de vue des valeurs véhiculées.

\section{BIBLIOGRAPHIE}

PESIKOVA, 1999 = ПЕСИКОВА АГРАФЁНА СЕМЁНОВНА, «ИСТОРИЯ СОЗДАНИЯ ШРИФТА ДЛЯ СУРГУТСКОГО ДИАЛЕКТА ХАНТЫЙСКОГО ЯЗЫКА», ХАНТЫ ЯСАНГ, http://www.khanty-yasang.ru/ article/65 (consulté le 2 février 2017).

TEREŠKIN, 1958 = ТЕРЁШКИН НИКОЛАЙ ИВАНОВИЧ, О НЕКОТОРЫХ ОСОБЕННОСТЯХ ВАХОВСКОГО, СУРГУТСКОГО И КАЗЫМСКОГО ДИАЛЕКТОВ ХАНТЫЙСКОГО ЯЗЫКА, ЛЕНИНГРАД.

TEREŠKIN, 1959 = ТЕРЁШКИН НИКОЛАЙ ИВАНОВИЧ, БУКВАРЬ ДЛЯ ПОДГОТОВИТЕЛЬНОГО КЛАССА ХАНТЫЙСКОЙ НАЧАЛЬНОЙ ШКОЛЫ (НА ЯЗЫКЕ СУРГУТСКИХ ХАНТЫ), ЛЕНИНГРАД.

TEREŠKIN, 1959 = ТЕРЁШКИН НИКОЛАЙ ИВАНОВИЧ, ПЕРЕВОД БУКВАРЯ НА ЯЗЫКЕ СУРГУТСКИХ ХАНТЫ ДЛЯ ПОДГОТОВИТЕЛЬНОГО КЛАССА ХАНТЫЙСКОЙ (СУРГУТСКОЙ) НАЧАЛЬНОЙ ШКОЛЫ, ЛЕНИНГРАД. TEREŠKIN, 1966 = ТЕРЁшКИН НИКОЛАЙ ИВАНОВИЧ, ХАНТЫЙСКИЙ ЯЗЫК, МОСКВА. TEREŠKIN, 1975 = ТЕРЁШКИН НИКОЛАЙ ИВАНОВИЧ, УЧЕБНИК ДЛЯ ПОДГОТОВИТЕЛЬНОГО КЛАССА (НА ЯЗЫКЕ СУРГУТСКИХ ХАНТЫ), ЛЕНИНГРАД.

TEREŠKIN, 1975 = ТЕРЁшКИН НИКОЛАЙ ИВАНОВИЧ, БУКВАРЬ ДЛЯ ПОДГОТОВИТЕЛЬНОГО КЛАССА (НА ЯЗЫКЕ СУРГУТСКИХ ХАНТЫ), ИЗДАНИЕ 2-Е, ИСПРАВЛЕННОЕ И ДОПОЛНЕННОЕ, ЛЕНИНГРАД. TEREŠKIN, 1981 = ТЕРЁШКИН НИКОЛАЙ ИВАНОВИЧ, СЛОВАРЬ ВОСТОЧНОХАНТЫЙСКИХ ДИАЛЕКТОВ, ЛЕНИНГРАД.

\section{NOTES}

1. En khanty : ХӐнты ЯС̆̆Ң.

\section{RÉSUMÉS}

Le khanty de Surgut, l'un des dialectes du khanty oriental, s'écrit depuis que N.I. Tereškin, en 1959, lui a donné un alphabet. Celui-ci est discuté dans le détail dans cet article, qui intervient dans le débat actuel sur l'écriture du khanty oriental et commente quelques traits caractéristiques de l'usage de cet alphabet aujourd'hui. 
Surgut Khanty is one of the Eastern Khanty dialects. It has been written since N.I. Terješkin, in 1959, invented an alphabet for it. This article discusses this alphabet in detail and takes stance in the debate about it. Moreover it comments upon some features of nowadays use of written language.

Surguti handi keel, mis on üks idahandi murretest, sai kirjakeeleks 1959. aastal, kui Terjoškin lõi sellele keelele tähestiku. Käesolevas artiklis vaadeldakse Surguti handi kirjakeele kasutust tänapäeval ning võetakse seisukoht selle üle toimuvates aruteludes.

\section{INDEX}

Thèmes : linguistique, phonologie

Index géographique : Agan, Jugan, Kazym, Neftejugansk, Nižnevartovsk, Pim, Surgut, Tromjogan, Vah, Vasjugan

Mots-clés : système graphique, système graphique, alphabet, alphabet, orthographe, orthographe, khanty de Surgut, khanty de Surgut

motsclesru ПИСЬМЕННОСТЬ, АЛФАВИТ, ОРФОГРАФИЯ, СУРГУТСКИЙ ХАНТЫЙСКИЙ ЯЗЫК

motscleset kirjasüsteem, tähestik, õigekiri, Surguti handi

Index chronologique : XXe siècle, XXIe siècle

Keywords : graphic system, alphabet, orthography, Surgut Khanty

nomsmotscles Khantys orientaux

disciplines khanty du Kazym, khanty du Nord, khanty de Surgut, khanty de Šuryškar, russe 